\title{
Clinical, and Diagnostic Characteristics of an Unsuspected Course of Urinary Tuberculosis: A Brief Report
}

\author{
Gloria G Guerrero ${ }^{1 *}$, Luis A Aguilera-Galaviz ${ }^{2}$, Arturo Araujo-Conejo ${ }^{3}$, \\ Iskra Tuero ${ }^{4}$ and Juan Manuel Favela-Hernández ${ }^{5,6}$ \\ ${ }^{1}$ Universidad Autónoma de Zacatecas, Unidad Académica de Ciencias Biológicas, \\ Zacatecas, Zac, México \\ ${ }^{2}$ Universidad Autónoma de Zacatecas Unidad Académica de Odontología, Programa de \\ Licenciatura de Médico Cirujano Dentista, Especialidad en Odontopediatría y Maestría \\ en Ciencias Biomédicas. Zacatecas, Zac, México \\ ${ }^{3}$ Hospital General de Zacatecas, Departamento de Investigación de los Servicios de \\ Salud (SSZ), Ciudad Gobierno, Zacatecas, Zac, México \\ ${ }^{4}$ Facultad de Ciencias y Filosofía, Universidad Peruana Cayetano Heredia, Lima-Perú \\ ${ }^{5}$ Universidad Juárez del Estado de Durango, Facultad de Ciencias Químicas, Gómez, \\ Palacio, Dgo, México \\ ${ }^{6}$ Instituto Multidisciplinario de Ciencias AVICENA, Campus Torreón, Torreón, Coahuila, \\ México \\ *Corresponding Author: Gloria G Guerrero, Universidad Autónoma de Zacatecas, \\ Unidad Académica de Ciencias Biológicas, Zacatecas, Zac, México.
}

Received: December 17, 2021

Published: January 21, 2022

(C) All rights are reserved by Gloria G

Guerrero., et al.

\begin{abstract}
Human Tuberculosis caused by Mycobacterium tuberculosis (MTb) remains a serious public health problem because of the high percentage of asymptomatic infection. Even worse, from this percentage, around $20 \%$ develop disseminated Tuberculosis involving the urinary tract. How to approach this?. A key issue is the accuracy and speediness of the diagnosis. In the present brief report, we described and pinpointed an unsuspected course of urinary Tuberculosis in an individual that presented signs not very characteristic of non pulmonary TB. The main symptoms were microhematuria, urinary incontinence, and urinary infections. To gain insight into the characterization of the clinical manifestations of the infectious disease, we pursued unconventional analysis assays, along with classic microbiological media tools. Molecular (microarrays, pattern of biomarkers) and serological reactivity (mycobacterial antigens detection) were determined in both blood and exudates. Remarkably, in the urinary diagnosis of Tuberculosis, these alternative tools could provide strong support and input to the clinics, especially in unsuspected cases.
\end{abstract}

Keywords: Urinary Tuberculosis; Hematuria; Urinary Contingency; BAAR; PCR; RT-PCR; Microarrays; Serological Reactivity

\section{Introduction}

Mycobacterium tuberculosis (MTb) is the etiological agent of Human Tuberculosis. It is a member of the Mycobacteriaceae family. Accordingly to the World Organization of Health (WHO), 1.7 million deaths and morbidity of 10.6 million. While most infected people remain asymptomatic (latent infection), only five percent develop the active disease [1,2]. Several factors favored the persistence and the global spread of MTb infection in developed countries as multi-drug drug-resistant strains (MDR), genetic susceptibility to mycobacterial infections, and other co-infections (HIV) [3-5]. 
Mycobacterium bovis Bacille-Calmette-Güerin (BCG) remains the only official vaccine worldwide effective against some forms of disseminated tuberculosis or tuberculosis associated with meningitis in children [6]. However, it fails to protect young and adult people [7].

Therefore, a much better understanding of the cellular and innate immune response protective antimycobacterial to facilitate the development of more efficacious vaccines against Mycobacterium tuberculosis as well as diagnostic or prognostic tests [8-12].

One form of extrapulmonary Tuberculosis that has been receiving attention is urinary tract Tuberculosis. It has become the third most common of disseminated Tuberculosis. Several studies have shown that $M$. tuberculosis can infect and disseminated toward other organs (urinary tract) [13-19]. In fact, from the 10 million of MTb infected people that develop pulmonary Tuberculosis, a higher of $20 \%$ develop genitourinary Tuberculosis affecting the anatomy and physiology of the urinary tract of men and women (UG-TB) [18-20]. Nowadays, IG-TB has become a serious and common health problem, especially in developed countries. The dormant state of the tubercle bacilli represents, thus, a "hot spot of infection" nowhere. The diagnostic of UG-TB is no easy task, since due precisely to the lack of clinic manifestations and the paucibacillary bacilli (1 to 9 BAAR X 100) [20,21]. The clinical manifestations of UG-TB are diverse. However, it is also true that co-morbility dity have also a role in the course and development of the infectious disease $[17,19,22,23]$.

How early are UG-TB diagnoses in an asymptomatic patient? It is almost imposible without imagenology to detect any changes in the urinary tract renal parenchyma.

However, radiological changes become more evident in the renal parenchyma as the disease progress $[19,22,24]$. The UG-TB diagnostic is essential for good renal and urinary tract function and to avoid severe complications. Renal TB diagnosis is not always easy because Mycobacterium tuberculosis (MTb) detection is not always possible (1 to 9 BAAR x 100). In addition, antibiotic treatment, early kidney damages, and rare urological diseases can mask infectious diseases. Therefore, the timing in the diagnostic of UGTB is fundamental [19,22,24-27].

\section{Molecular techniques}

Since polymerase chain reaction (PCR) discovering by Kary Mullis in 1983 becomes the molecular method for amplification to a small and large scale of DNA genomic sequence. More important outstanding were amplifying in samples with a low number of bacilli. The use of PCR in GUTB diagnostic was not the exception. Thus, van Vollenhoven in 1996 [28] validated the use of PCR from concentrated urine, resulting in faster than microbiological urine culture [16]. Despite this progress in UG-TB diagnosis, lesions by careful radiological examination in individuals of Africa and Europe [28,30]. Diagnosis of tuberculosis of renal and urinary tract using two methodologies. The identification of Koch acid-fast bacilli Ziehl-Nielsen stained on renal parenchyma biopsies and upper urinary tract [31]. Recent reports propose UG-TB diagnosis on three parameters: case history, pyuria, and radiological imaging. Other authors have suggested that histochemistry combined with molecular assays provide a more precise, accurate, and definitive UG-TB [18,27,31-36].

\section{Microbiological techniques}

One of the best biological diagnostic tests is the detection of the bacillus in clinical sample, be any fluids (urine, sputum) or tissue [23,36-39]. It is usually called as the gold microbiological test for UG-TB.

\section{Imaging techniques}

Imaging techniques have made it possible to localize sites and spread disease. Thus, determining the effect of the infectious lesion damage and associated pathology. In addition, using imaging equipment is also possible to have an alternative to obtain samples of the tissues or organ involved [23,38,40]. Furthermore, a set of integrated techniques yields good and faster results $[41,42]$.

Biomarkers determination leads to a better understanding of the components that determine the disease spectrum, the reactivation of the dormant mycobacteria, and the progression of the disease [31,36,43-48]. Therefore, this tool represents a powerful and potential alternative to predict or define prognostic or diagnostic tests. Microarrays technologies have enabled biomarkers candidates determination to differentiate active versus latent Mycobacterium tuberculosis infection [47-48]. So far, this could potentiate the sensibility and specificity of diagnostic and prognostic

Citation: Gloria G Guerrero., et al. "Clinical, and Diagnostic Characteristics of an Unsuspected Course of Urinary Tuberculosis: A Brief Report". Acta Scientific Microbiology 5.2 (2022): 48-55. 
tests. However, microarrays still need to be scaled and developed for clinical purposes. Therefore, molecular methods that include amplification of mycobacterial DNA [34;49]; RT-PCR, GeneXpert MTB/RIF assay [27,35]. A series of methodologies can help in the diagnosis of urinary TB [23]. With the remarkable breakthroughs of the Next-Generation Sequencing (NGS) development, it would be expecting as a promising molecular tool for UG-TB diagnostic [37]. The challenge of this: be able to develop less invasive techniques, specific, sensitive diagnostic tests [26], and to have sufficient information to predict progression. Therefore, the goal for a successful UG-TB treatment outcome: an early, fast, and accurate diagnosis to avoid disease progression and kidney organ failure[23,26].

\section{Clinical, Diagnostic characteristics}

The clinical symptoms that an individual presented were incontinence, microhematuria, and recurrent infections in the urinary tract. Allergic to Ceftriaxone, penicillin, sulpha, and metoclopramide non-steroidal anti-inflammatory drugs (NSAD).Tolerant to amikacin and phosphocyl. Septumplasita SII in Torax. No systemic arterial hypertension (SAH). No Diabetes Mellitus type two) (DM2). No distioidismo (DT). Allergic to Ceftriaxone, penicillin, sulpha, and metoclopramide non-steroidal anti-inflammatory drugs (NSAD).Tolerant to amikacin and phosphocyl. No blood transfusion (BT), neither alcoholism nor smoking. Blood tests as a normal routine laboratory. Laboratory investigations were performed and revealed normal kidney function with a creatinine of $61 \mu \mathrm{mol} / \mathrm{L}$, urea 30.4, HBA x 6.58, hb 14,4; platelets, 338000 per microliter (mcL) (normal value of 150,000 a 400,000 per microliter (mcL) or (150 a $400 \times 109 / \mathrm{L}$ ); leucocytes, 6900 (normal values under 10.000 cells per $\mathrm{mL}$ ). Uroculture: Escherichia coli multisensible to antibiotics. Some GUSE (general urine sediment exam) with hematuria gives the impression of the infection of urinary tracts (IDX) or recurrent infections of urinary tracts and urinary incontinence. Since the symptoms continue, the patient attends the infectology service unit, which recommends specialized laboratory tests to discard any sexual transmission disease and renal tuberculosis. It was performed urine cytology in serial urine of three for cytologic studies prepared for Papanicolau and Zhiel Nielsen staining (Macroscopic description). Negative for malignancy, abundant inflammatory cells, predominantly macrophages, and lymphocytes (+). Urothelial cells show tiny inflammatory reactive changes, Bacilar flora changes $(++)$, positive bacillus acid alcohol resistance (B.A.A.R.) (Zhiel Neelsen staining) for one of the samples.

A molecular test was applied. Real-time PCR confirmed the presence of M. tuberculosis. Next, the patient attends the Unit of Research of the Institute Mexican of Social Security (IMSS) that collects three serial urine. Both acid-alcohol-resistant bacilli (AARB) and GeneXpert were negative. The early Mycobacterium tuberculosis diagnoses revealed the presence of the mycobacteria. However, the patient continues with discomfort, microhematuria, and urine infections. Amikacin and phosphodyl recommend due to the patient's allergic to several antibiotics. After five months post-third's positive results except for the urinary incontinence, she attends Nephrology service. Further microbiological, immunological, and molecular tests confirmed the early MTb infection. According to the physical examination (PE) of the patient: Height $(1.71 \mathrm{~m})$, weight $(60 \mathrm{Kg})$, temperature $\left(36^{\circ} \mathrm{C}\right)$, arterial pressure $(130 / 80 \mathrm{mmHg})$; heart rate ( $80 / \mathrm{min})$, and breathing respiratory (20 resp/min). The patient was observed conscious, oriented, and hydrated, in normal oropharyngeal in CR RsCsRs no aggregations in normal pulmonary area, in the abdomen without compromise, no edemas, of Ms. Is. Physical Examination (PE); height (1.71 m), weight (60 Kg), glucose (ND), temperature (36oC). Arterial pressure (130/80 mmHg); heart rate (80 / $\mathrm{min})$; breathing respiratory (20 resp/min).

\section{Discussion and Conclusion}

Urinary tract infections are one of disseminated tuberculosis common in $20 \%$ of the individuals infected with Mycobacterium tuberculosis. Herein, a brief report of an unsuspected course of Urinary Tuberculosis. The individual from the infectology service. The clinical signs were not very characteristic of no pulmonary TB. An individual refers to urinary incontinence episodes, recurrent urinary infections, and suffering from intermittent stages of microscopic hematuria. Therefore, the initial diagnostic is to discard glomerulopathy and renal tuberculosis. The historical clinic of incontinence urinary requires the evaluation by Urology in the way to make a Chirurgie resolution if it is considered necessary. On nephrology does not require management. At this moment, and in such a case, it requires determining the origin of hematuria. New paraclinical tests on urinary sediment include seric creatinine and urea to evaluate renal function. 
Laboratory tests revealed normal kidney function with a creatinine of $61 \mu \mathrm{mol} / \mathrm{L}$, urea 30.4, hba x 6.58, hb 14,4. Platelets, 338000 per microliter (mcL) (normal value of 150,000 to 400,000 per microliter (MCL). Leucocytes 6900 (normal values under 10.000 cells per mL). Uroculture: Escherichia coli multisensible to antibiotics. Some GUSE (general urine sediment exam) with hematuria gives the impression of recurrent infections of urinary tracts and urinary incontinence. Since the discomfort continued, a detailed oncologic and pathological examination was carried out (Figure 1A-B). The results showed urine serial cytologic bacilar flora (++). Acid alcohol-resistant bacillus (BAAR) $(+)$ in one of the series samples. Abundant inflammatory cells, macrophages, and lymphocytes (+), urothelial cells present inflammatory changes reactive, bacilar flora (++). Zhiel Neelsen shows positivity in one of the samples. Molecular assay. RT-PCR in real-time of urine with beta-globin as controls. M. tuberculosis (+); M. bovis (-). 7500 Fast Real-Time PCR system; Applied BiosystemsTM. The adult individual was evaluated in infectology service by suspected renal tuberculosis, which discards with GenXpert and MGIT culture.

Since the discomfort persisted, a third diagnostic assay in the laboratory research investigation continued as outlined in Figure
1A-B). From the literature, it is well known that biomarkers determination leads to a better understanding of the components that determine the spectrum disease. The reactivation of the dormant mycobacteria, and the progression of the disease [36,43-48], providing thus, a feasible tool to predict or define prognostic or diagnostic tests [50-52]. These assays like tests based on several methodologies: Microbiological techniques, Serological mycobacterial antigen reactivity (Figure 1C; Figure 2) [53], Molecular tools (PCR) [54], and a set of a profile of up and down-regulated genes (i.e. genes that participate in autophagy, innate immue response, MAPKs, cellular transport)(Tables 1A-B). All of them, strengthened the data obtained by early real-time PCR and classical BAAR (Figure 1A-B). Indeed, in previous work, we proposed that these methodologies can be extrapolated to human Tuberculosis [54]. Moreover, Berry et al., 2010 [44] reported the blood transcriptional signature in active TB patients.

Collectively, by the data presented here, we can conclude that a combination of clinics and diagnosis leads to a shortcut in UG-TB diagnosis, providing hope and a better therapeutic intervention for patients with unsuspected course of Urinary Tuberculosis.

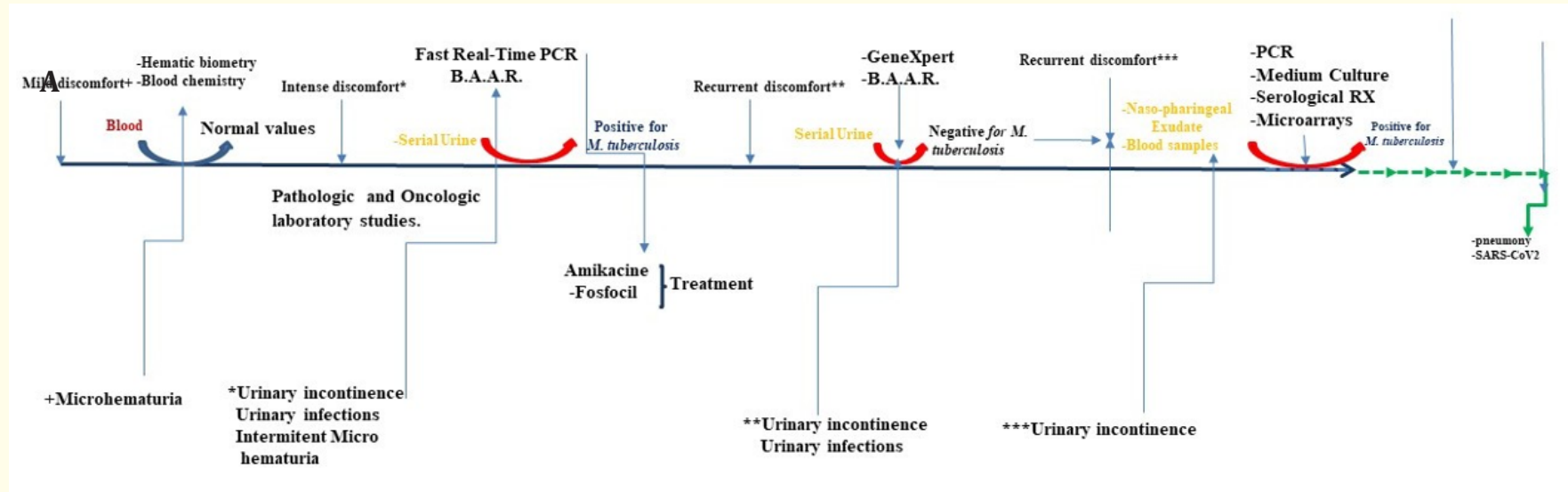

Figure 1. Outline of the clinic, and diagnostic characteristics of an unsuspected course of Urinary Tuberculosis. 
B

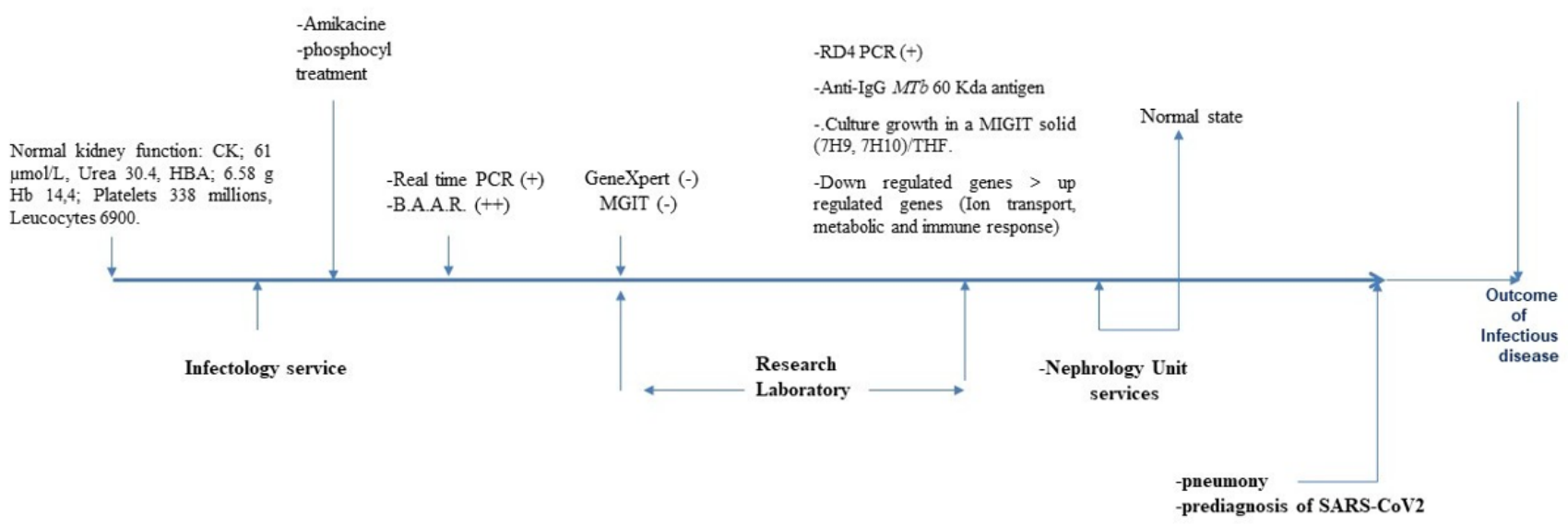

Figure 1. Clinical, and Diagnostic characteristics of an unsuspected course of Urinary Tuberculosis. A. Outline of the clinic and diagnostic characteristics of the patient. B. A summary of the classical (microbiology, biochemical), and no classical (serological reactivity toward mycobacterial antigens serology), molecular methodologies (PCR, real-time PCR, microarrays). C. Scheme of the different methodologies used, of paramount to reach UG-TB diagnostic.

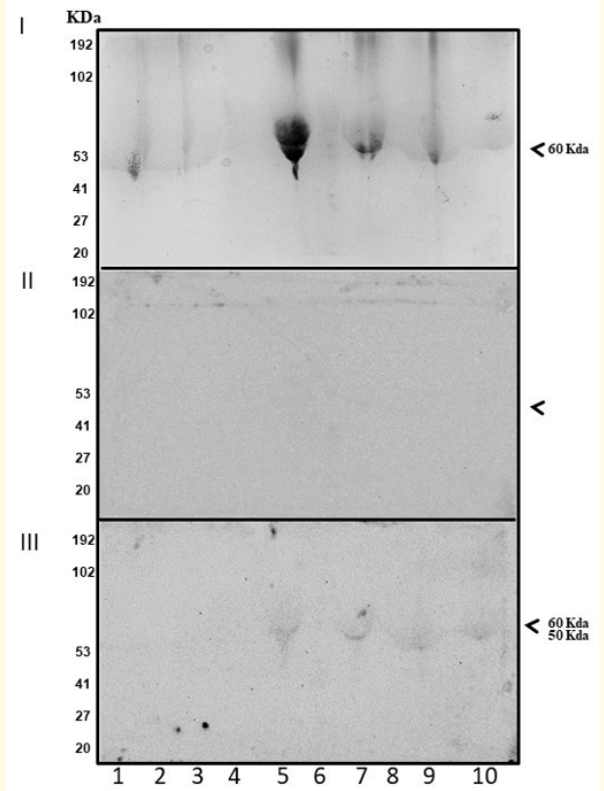

Figure 2. Serological reactivity of IgG antibodies to Mycobacterium tuberculosis (MTB) antigens's in an unsuspected course of Urinary Tuberculosis. MTB antigens of the complex of M. tuberculosis [(M. bovis BCG, M. bovis, and the M. bovis AN5 (reference strain)] were obtained as reported by Favela-Hernandez., et al. 2019. Herein, patients serum reacts predominantly toward a band of proteins of around 50-60 Kda (A). Lane 1: Molecular Weight Markers (MWM). Lane 2. M. bovis BCG. Lanes 3-4, M. tuberculosis attenuated (HA1). Lane 5, M. bovis East. Lane 6, empty. Lane 7, M. bovis AN5). Lane 8, empty. Lanes 9-10, M. tuberculosis H37Rv). 
A

\begin{tabular}{|c|c|c|c|c|c|}
\hline Code & Gene name & $\begin{array}{l}\text { QP fioli } \\
\text { changet }\end{array}$ & code & Gene name & $\begin{array}{l}\text { Down (fold } \\
\text { change) }\end{array}$ \\
\hline 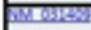 & Cot6 & & $2.41 N 0,005625$ & n.x.5 & 2.02 \\
\hline GM_02053: & vocisis & & 2.5es NU_005283 & ronos & -2058 \\
\hline Fim_001482 & satu & & 3.54 NU_Oosors & Vеам1 & .212 \\
\hline M_coos? & geas & & 2.: NM $00035 s$ & TGros & -210 \\
\hline M_cossse & $100 \times 2$ & & 2.874 NM 017874 & C.200rt27 & $-2.17 \%$ \\
\hline M_cos:s? & ance & & 2.086 NU_Cos985 & FNo: & -2224 \\
\hline Gm_0osssz & oras & & $2 . m$ Nu cosiss & coxis & -2.274 \\
\hline M_coss327 & nureser & & 2.761 NU 021252 & 2019:9 & -228 \\
\hline M_022369 & pusases & & 2.73 NU 013272 & SLCOBAI & -2382 \\
\hline M_coss56 & cot236 & & $2.563 \mathrm{NU}$ C03844 & FNFRSFIOA & -2391 \\
\hline TM_01835e & casert2 & & 2.513 NU_Co65068 & Tua6 & .2456 \\
\hline am_020062 & 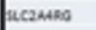 & & 2.241 NM 006866 & |uvas & -2093 \\
\hline Ty_orses & gesous & & 2.295 NU 006648 & hexWNac2 & .2526 \\
\hline M_c01152 & acasus & & $2.274 \mathrm{NU} 004172$ & secies & .256 \\
\hline TM_oovses & peoses & & 2.239 NU 004612 & tafbez & .2568 \\
\hline M_cosst2 & $\cos 20 / 1$ & & 2.233 NV_. 032986 & Cosps & -2.740 \\
\hline Tim_oss 228 & moncis & & 2.2 NU 006449 & COCA2EP3 & -286 \\
\hline Su_cosuss & torn & & 2.2.17 NM 025159 & Cxorf21 & -2002 \\
\hline M_co03242 & unarca & & 2.:51 NM 004512 & II12A & -3.363 \\
\hline 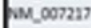 & Torece & & 2.2.69 NM 006564 & cxoa6 & -3.40 \\
\hline M_00425: & pocoso & & 2. 2007 AF284434 & 4.12:2 & -353 \\
\hline GM_013ass & consa & & 2.053 NM 002170 & IFAS & .4 .423 \\
\hline Sim_ormos & ESond4 & & 2.066 NM 019111 & HLA-DRA & -468 \\
\hline GM_002032 & puascin & & 2054 NM 004625 & WNT7A & .455 \\
\hline bаe onzsus & heares & & دарихисон & Lannosis & \\
\hline
\end{tabular}

B

\begin{tabular}{|c|c|c|c|}
\hline Pathway ( $\left.\mathrm{C}_{\mathrm{p}}\right)$ & symbol gene & fold change $p$ & \\
\hline Loss of function of TGF8R2 in cancer & TGFBR2 & 2.15 & 0.002 \\
\hline Loss of function of TGFBR1 in cancer & TGFBR2 & 2.15 & 0.004 \\
\hline TGF- $\beta$ receptor signaling in EMT & TGFBR2 & 2.15 & 0.017 \\
\hline Signaling by TGF-beta receptor & TGFBR2 & 2.15 & 0.024 \\
\hline SMAD2/3-phosphorylation motif mutants in cancer & TGFBR2 & 2.15 & 0.004 \\
\hline Loss of function of $\mathrm{SMAD} 3$ in cancer & TGFBR3 & 2.15 & 0.024 \\
\hline IRAK4 deficiency(TLR2/4) & IRAK2 & 2.6 & 0.014 \\
\hline Trafficking of GLUR2 containing AMPA receptors & COK2AP1 & 2.23 & 0.019 \\
\hline HSF1 astivation & HSS2 & 2.3 & 0.05 \\
\hline Pathway (dowa) ) & symbol gene & fold change $P$ & \\
\hline Loss of function of TGFBR2 in cagcer & TGFB1 & \multicolumn{2}{|c|}{$-2.172,28 \times 10-5$} \\
\hline Loss of function of TGFBR1 in cancer & TGFBR1 & \multicolumn{2}{|c|}{$-2.576 .96 \times 10.5$} \\
\hline TGF- $\beta$ receptor signaling in EMT & TGFBR1 & -2.57 & 0.001 \\
\hline Signaling by TGF- $\beta$ receptor & TGFB1 & -2.17 & 0.002 \\
\hline Downregulation of TGF- $\beta$ receptor signaling & TG5B1 & -2.13 & 0.008 \\
\hline Dimerization of procaspase 8 & TNFRSE $10 \mathrm{~A}$ & -2.39 & 0.002 \\
\hline Regulation of cFUP & TNFRSF $10 \mathrm{~A}$ & -2.39 & 0.051 \\
\hline IRAK4 deficiencly (TLR 2/4) & IRAK2 & -2.46 & 0.077 \\
\hline Il-36 pathway & IL1RL2 & -2.13 & 0.043 \\
\hline
\end{tabular}

The pattern of up and domn regulated senes were determined using DNA microarray. eDNA was prepared from RNA using the KIT (first-strand eDNA labeling KIT (lmitrogen). $M$. mblerewlosis DNA Microarrays prepared and hybridized with eDNA's pasiest of bealty individual (A)(Unit of Microarray, IFC-UNAM, Mexico Cay). The microarray analysis using genarise. Up and Down-regulated geses ther for two cutots, oee for Z-Score ligher than 1.5 and the secoed, with $Z$-Sceres hipher than 2 (> 1.5 and 2.0 SD) (P< 0.05 ) while Graptipad

7.0 program software for beat maps anslysis (A). Reactome functional anslysis (B) based co the probabăty (p vabse) that a reaction ocer in a pathway (Fabrezar et al, 2017).

\section{Acknowledgements}

We are in debt with Dr Myriam Bobadilla (INCMSZ; Mexico) for providing Mycobacterium bovis AN5 strain. We thanks to the Unit of Microarrays from the IFC-UNAM. Mexico City. Mexico.

\section{Ethics Approval and Consent to Participate}

The study and all the procedures for medical research involving human subjects, including research on identifiable human material and data were performed under the principles of the Declaration of Helsinski, and approved by the ethic commitee in Research of the Zacatecas, General Hospital "Luz Gonzalez Cosio" CONBIOETICA32-CEI-001-20180807.

\section{Consent for Publication}

Written informed consent was obtained from the patient for publication of this case report and any accompanying images. A copy of the written consent is available for review.

\section{Competing Interests}

The authors declare no competing of interests.

\section{Availability of Data and Material}

Data will be shared following institutional guidelines. The review of the literature was based on search and data from Pubmed database without limitation to 2021.

\section{Funding}

The study did not receive funding from any dependence nor a grant. G.G.G.M. and J.M.F.H. received a fellowship by the National System of Researchers (SNI-CONACYT). Mexico. G.G.G.M. is Perfil Prodep (A program of the National Secretary of Education).

\section{Author's Contributions}

G.G.G.M. conceptualization, methodology, analysis and writing. L.A.A.G. collaboration in patient's contact, discussion. A.A.C.; I.T. and J.M.F.H. Reviewing of the report. All authors have read and approved the manuscript.

\section{Bibliography}

1. World Health Organization (WHO). "Global Tuberculosis control; surveillance, planning financing". WHO/HTM/ TB/2008.393. Geneva, Switzerland: WHO (2010a). 
2. World Health Organization (WHO). "Treatment of Tuberculosis: Guidelines". $4^{\text {th }}$ ed. WHO (online) (2010b).

3. Maher D and Raviglione M. "Global epidemiology of Tuberculosis”. Clinics in Chest Medicine 26 (2005): 167-182.

4. Hershberg R., et al. "High functional diversity in M. tuberculosis driven by the genetic drift and human demography". PloS Biology 6 (2008): e311.

5. Young DB., et al. "Confronting the scientific obstacle to global control of Tuberculosis". Journal of Clinical Investigation 118 (2008): 1255-126.

6. Rodríguez LC., et al. "Protective effect of BCG against Meningitis and Miliary Tuberculosis: a Meta-analysis". International Journal of Epidemiology 22 (1993): 1154-1158.

7. Fine PEM. "Variation in protection by BCG: implications of and for heterologous immunity". Lancet 346 (1995): 1339-1345.

8. Herrera V., et al. "Clinical application and limitations of interferon-gamma release assays for the diagnosis of latent tuberculosis infection". Clinical Infectious Diseases 52 (2011): 10311037.

9. Abebe F. "Is Interferon-gamma the right marker for Bacille Calmette-Guerin-induced immune protection? The missing link in our understanding of tuberculosis". Clinical and Experimental Immunology 169 (2012): 213-219.

10. Koul A., et al. "Interplay between mycobacteria and host signaling pathways". Nature Reviews Microbiology 2 (2004): 189202.

11. Pulendran B and Ahmed R. "Translating innate immunity not immunological memory: implications for vaccine development". Cell 124 (2006): 849-863.

12. Mohr I and Sonenberg N. "Host translation of the nexus of infection and immunity". Cell Host Microbe 12 (2012): 470-483.

13. WHO. "Guidelines for the treatment of drug-susceptible tuberculosis and patient care" (2017).

14. WHO. "WHO Global Tuberculosis report 2018” (2018).

15. WHO. "The top 10 causes of death" (2019).

16. Wise GJ. "Urinary tuberculosis: modern issues". Current Urology Reports 10 (2009): 313-318.

17. Figueiredo AA., et al. "Epidemiology of urogenital tuberculosis worldwide". International Journal of Urology 15 (2008): 827832.
18. Abbara A., et al. "Etiology and management of genitourinary tuberculosis". Nature Reviews Urology 8 (2011): 678-688.

19. Floyd K., et al. "The global tuberculosis epidemic and progress in care, prevention, and research: an overview in year 3 of the End TB Was". Lancet Respiratory Medicine 6 (2018): 299-314.

20. Wise GJ and Shteynshlyuger A. "An update on lower urinary tract tuberculosis". Current Urology Report 9 (2008): 305-313.

21. Adhikari S and Basnyat B. "Extrapulmonary tuberculosis: a debilitating and often neglected public health problem”. BMJ Case Report 11 (2018): e226098.

22. Yadav S., et al. "Genital tuberculosis: current status of diagnosis and management". Translational Andrology and Urology 6 (2017): 222-233.

23. Munner A., et al. "Urogenital tuberculosis - epidemiology, pathogenesis and clinical features". Nature Reviews Urology 16.10 (2019): 573-559.

24. Kulchavenya E., et al. "Urogenital tuberculosis: classification, diagnosis, and treatment". European Urology 15 (2016): 112121.

25. Liu HY., et al. "Tuberculous epididymitis: a case report and literature review". Asian Journal of Andrology 7 (2005): 329-332.

26. Mert A., et al. "Urinary tuberculosis: still a challenge". World Journal of Urology 38 (2020): 2693-2698.

27. McNerney R and Zumla A. "Impact of the Xpert MTB/ RIF diagnostic test for tuberculosis in countries with a high burden of disease". Current Opinion in Pulmonary Medicine 21 (2015): 304-308.

28. van Vollenhoven P., et al. "Polymerase chain reaction in the diagnosis of urinary tract tuberculosis". Urology Research 24 (1996): 107-111.

29. Dochviri TZ., et al. "Detection of mycobacteria tuberculosis in patients with urogenital tuberculosis by PCR method". Georgian Medical News 119 (2005): 14-17.

30. Valentini AL., et al. "Diagnostic imaging of genitourinary tuberculosis". Rays 23 (1998): 126-143.

31. Traşcă E., et al. "The place and the role of histological examination in diagnostic algorithm of urinary system tuberculosis". Romanian Journal of Morphology and Embryology 46 (2005): 105-108. 
32. Hemal AK., et al. "Polymerase chain reaction in clinically suspected genitourinary tuberculosis: comparison with intravenous urography, bladder biopsy, and urine acid fast bacilli culture". Urology 56 (2000): 570-574.

33. Lenk S. "Genitourinary tuberculosis in Germany: diagnosis and treatment". Urology 50 (20115): 1619-1627.

34. Sanchez I., et al. "Urinary Tuberculosis: Serious Complications May Occur when Diagnosis is Delayed". Acta Médica Portuguesa 28 (2015): 382-385.

35. Lawn SD., et al. "Advances in tuberculosis diagnostics: the Xpert MTB/RIF assay and future prospects for a point- 353 ofcare test". Lancet Infectious Disease 13 (2013): 349-361.

36. Walzi G., et al. "Immunological biomarkers of tuberculosis". Nature Reviews Immunology 11 (2011): 343-354.

37. WHO. "The use of next-generation sequencing technologies for the detection of mutations associated with drug resistance in Mycobacterium tuberculosis complex: technical guide" (2018).

38. Bhalla M., et al. "Performance of light-emitting diode fluorescence microscope for diagnosis of tuberculosis. International Journal of Mycobacteriology 2 (2013): 174-178.

39. Lawn SD and Zumla AI. "Tuberculosis (Seminar)". Lancet 378 (2011): 57-72.

40. Davis JL., et al. "Diagnostic accuracy of same-day microscopy versus standard microscopy for pulmonary tuberculosis: a systematic review and meta-analysis". Lancet Infectious Disease 13 (2014): 147-154.

41. Zajaczkowski T. "Genitourinary tuberculosis: historical and basic science review: past and present". Central European Journal of Urology 65 (2012): 182-187.

42. Shevchenko SY and Kulchavenya EV. "Is there screening for urinary tuberculosis?”. Urologia (2017): 34-37.

43. Cheng VC., et al. "Molecular diagnostics in tuberculosis". European Journal of Clinical Microbiology and Infectious Diseases 24 (2005): 711-720.

44. Berry PRM., et al. "An interferon-inducible neutrophil-driven blood transcriptional signature in human tuberculosis". Letter to Nature 466 (2010): 973-979.

45. Mistry R., et al. "Gene expression patterns in whole blood identify subjects at risk for recurrent tuberculosis". Journal of Infectious Disease 195 (2007): 357-365.
46. Ahmad S. "Pathogenesis, immunology and diagnosis of latent Mycobacterium tuberculosis infection". Clinical and Developmental Immunology (2011): 814943- 814960.

47. Shu CC., et al. "Apoptosis associated biomarkers in tuberculosis: promising for diagnosis an prognosis prediction". BMC Infectious Disease 13 (2013): 45.

48. Whitworth SH., et al. "Biomarkers of tuberculosis: a research roadmap”. Biomark Medicine 7 (2013): 349-362.

49. Kraemer ZC., et al. "Complete genome sequencing of Mycobacterium bovis SP38 and comparative genomics of Mycobacterium bovis and M. tuberculosis strains". Frontiers in Microbiology 8 (2017): 1-10.

50. Mortazavi A., et al. "Mapping and quantifying mammalian transcriptomes by RNA-seq". Nature Methods 5 (2008): 621-628.

51. Malone JH and Oliver B. "Microarrays, deep sequencing and the true measure of the transcriptome". BMC Biology 9 (2011): 34-43.

52. Fabregat A., et al. "Reactome pahway analysis: A high performance in memory approach". BMC Bioinformatics 18 (2017): 142.

53. Favela-Hernández JM., et al. "Evaluation of Mycobacterium bovis isolated from cattle in Mexico for serum reactivity and antigen production kinetics". Journal of Medical Microbiology and Diagnoses 8 (2019): 1-6.

54. Sánchez-Garza JJ., et al. "Direct DNA modified CTAB preparation from nasal exudate in live M. bovis infected cattle in Mexico provide with a valuable assay extrapolated to humans TB diagnostic test". Journal of Tropical Diseases and Public Health 7 (2019): 1-7.

\section{Assets from publication with us}

- Prompt Acknowledgement after receiving the article

- Thorough Double blinded peer review

- Rapid Publication

- Issue of Publication Certificate

- High visibility of your Published work

Website: www.actascientific.com/

Submit Article: www.actascientific.com/submission.php

Email us: editor@actascientific.com

Contact us: +919182824667 